\title{
Complexity of compatible measurements
}

\author{
Paul Skrzypczyk $\odot,{ }^{1}$ Matty J. Hoban $\odot,^{2}$ Ana Belén Sainz $\odot,{ }^{3,4}$ and Noah Linden ${ }^{5}$ \\ ${ }^{1}$ H. H. Wills Physics Laboratory, University of Bristol, Tyndall Avenue, Bristol BS8 1TL, United Kingdom \\ ${ }^{2}$ Department of Computing, Goldsmiths, University of London, New Cross, London SE14 6NW, United Kingdom \\ ${ }^{3}$ International Centre for Theory of Quantum Technologies, University of Gdańsk, 80-308 Gdańsk, Poland \\ ${ }^{4}$ Perimeter Institute for Theoretical Physics, 31 Caroline Street N, Waterloo, Ontario, Canada N2L $2 Y 5$ \\ ${ }^{5}$ School of Mathematics, University of Bristol, University Walk, Bristol BS8 1TW, United Kingdom
}

(Received 16 October 2019; accepted 14 May 2020; published 5 June 2020)

\begin{abstract}
Measurement incompatibility is one of the basic aspects of quantum theory. Here we study the structure of the set of compatible, i.e., jointly measurable, measurements. We are interested in whether or not there exist compatible measurements whose parent is maximally complex, in the sense of requiring a number of outcomes exponential in the number of measurements, and related questions. Although we show this to be the case in a number of simple scenarios, we show that generically it cannot happen, by proving an upper bound on the number of outcomes of a parent measurement that is linear in the number of compatible measurements. We discuss why this does not trivialize the problem of finding parent measurements, but rather shows that a trade-off between memory and time can be achieved. Finally, we also investigate the complexity of extremal compatible measurements in regimes where our bound is not tight and uncover rich structure.
\end{abstract}

DOI: 10.1103/PhysRevResearch.2.023292

\section{INTRODUCTION}

One of the first lessons one typically learns about quantum mechanics is that, in general, observables do not commute and that this leads, for example, to the famous uncertainty principle [1]. The noncommutativity of observables shows that not all properties of a system can be measured simultaneously in quantum theory. Only when observables commute does a measurement in their common eigenbasis allow for the outcome of both measurement to be obtained simultaneously. The lack of commutativity is an indication that, in general, measurements are incompatible.

From a modern perspective, not all measurements are projective and commutativity no longer fully captures the notion of incompatibility. In particular, any set of positive operators $\mathbb{M}=\left\{M_{a}\right\}_{a}$ that sum to the identity $\sum_{a} M_{a}=\mathbb{1}$ constitutes a valid measurement, known as a positive-operator-valued measure (POVM) measurement. Although for POVMs there are multiple notions of compatibility, the most important is the notion of joint measurability $[2,3]$. This concerns whether there exists a single parent measurement which can be measured in place of the individual measurements and can be used to determine all their outcomes.

Parent measurements are in general more complex than their children. In particular, they are more complex in the sense of generally having many more outcomes than their children. Our interest here is in how complex parent measurements need to be in general, i.e., in just how many outcomes

Published by the American Physical Society under the terms of the Creative Commons Attribution 4.0 International license. Further distribution of this work must maintain attribution to the author(s) and the published article's title, journal citation, and DOI. they need to have. While it is known that parents need to have at most a number of outcomes that is exponential in the number of measurements, it is not known to what extent this bound is tight. The exponential number of outcomes makes determining whether a set of measurements is compatible, and finding a parent, difficult. In this paper we instigate the study of this and related questions. These questions probe the structure of the set of compatible quantum measurements, a topic which has to date received very little attention $[4,5]$ (see also [6,7] for related structural questions about individual POVMs). We give examples of measurements whose parents are maximally complex; however, our main result is to find an upper bound on the complexity of parent measurements. Our bound, which is linear in the number of measurements, is generically significantly tighter than the exponential bound. We show nevertheless why the problem of determining whether a set of measurements is compatible, and finding the corresponding parent, remains difficult even in light of our bound.

\section{JOINT MEASURABILITY}

Consider a pair of POVM measurements $\mathbb{M}=\left\{M_{a}\right\}_{a}$ and $\mathbb{N}=\left\{N_{b}\right\}_{b}$, with $o$ outcomes each. This pair of measurements is jointly measurable if there exists a measurement $\mathbb{K}=\left\{K_{\lambda}\right\}_{\lambda}$ with $k$ outcomes, $\lambda \in[k] \equiv\{1, \ldots, k\}$, and a pair of partitions of $[k]$ (nonempty disjoint subsets), $\left(P_{a}^{\mathbb{M}}\right)_{a}$ and $\left(P_{b}^{\mathbb{N}}\right)_{b}$, such that

$$
M_{a}=\sum_{\lambda \in P_{a}^{\mathbb{M}}} K_{\lambda}, \quad N_{b}=\sum_{\lambda \in P_{b}^{\mathbb{N}}} K_{\lambda} .
$$

That is, instead of measuring $\mathbb{M}$ or $\mathbb{N}$ individually, the measurement $\mathbb{K}$ can be performed and the outcomes $a$ and $b$ generated purely classically as a function of the outcome $\lambda$. 
In (1) each POVM element of $\mathbb{M}$ and $\mathbb{N}$ is a sum of POVM elements of $\mathbb{K}$. For each value of $\lambda$ we can specify which outcome $a$ and $b$ occurs for $\mathbb{M}$ and $\mathbb{N}$, respectively. This shows that there is a canonical form of parent measurement $\mathbb{C}=$ $\left\{C_{a b}\right\}_{a, b}$ which has $o^{2}$ outcomes such that $C_{a b}$ is associated with the outcome $a$ for $\mathbb{M}$ and $b$ for $\mathbb{N}$, i.e., such that

$$
M_{a}=\sum_{b} C_{a b}, \quad N_{b}=\sum_{a} C_{a b} .
$$

This highlights that we never need to consider parent measurements with more than $o^{2}$ outcomes, since if two elements have the same label $a b$ when put into canonical form, they are simply added together.

More generally, a collection of $m$ measurements $\left\{\mathbb{M}_{x}\right\}_{x}$, indexed by $x$, such that $\mathbb{M}_{x}=\left\{M_{a_{x} \mid x}\right\}_{a_{x}}$, is jointly measurable if there exists a parent $\mathbb{C}=\left\{C_{\mathbf{a}}\right\}_{\mathbf{a}}$, with outcomes indexed by the tuple $\mathbf{a}=\left(a_{1}, a_{2}, \ldots, a_{m}\right)$, such that

$$
M_{a_{x} \mid x}=\sum_{\mathbf{a} / a_{x}} C_{\mathbf{a}},
$$

where $\mathbf{a} / a_{x}=\left(a_{1}, \ldots, a_{x-1}, a_{x+1}, \ldots, a_{m}\right)$. That is, the children now arise by marginalizing over all but one of the outcomes of the parent.

One notable implication of the canonical form is that deciding whether a set of measurements is compatible is an instance of semidefinite programming (SDP) [8], since the conditions (3) that ensure $\mathbb{C}$ is a parent measurement are linear equality constraints, which need to be imposed in addition to the normalization and positivity conditions that ensure $\mathbb{C}$ is a valid POVM. In particular, it is the following feasibility SDP:

$$
\begin{aligned}
\text { find }\left\{C_{\mathbf{a}}\right\}_{\mathbf{a}} & \\
\text { subject to } M_{a \mid x} & =\sum_{\mathbf{a}} D_{\mathbf{a}}(a \mid x) C_{\mathbf{a}} \forall a, x, \\
C_{\mathbf{a}} & \geqslant 0 \forall \mathbf{a}, \\
\mathbb{1} & =\sum_{\mathbf{a}} C_{\mathbf{a}} .
\end{aligned}
$$

Here $D_{\mathbf{a}}(a \mid x)=\delta_{a, \mathrm{a}_{x}}$ can be thought of as a collection of deterministic conditional probability distributions, labeled by a, such that $a=\mathrm{a}_{x}$ with certainty. As we will discuss next, the canonical form also has implications for the complexity of joint measurability.

\section{THE COMPLEXITY OF COMPATIBILITY}

An important first observation is that there is an upper bound on the number of outcomes that a parent measurement needs to have, if it exists. In particular, every parent can be written in canonical form, with outcomes labeled by the tuple a. If each of the measurements $\mathbb{M}_{x}$ has $o$ outcomes, then the there are only $o^{m}$ tuples, and hence only $o^{m}$ outcomes.

At first sight, it might appear that parents are inherently more complex than their children, having a number of outcomes that is exponential in the number of measurements. There are two reasons why this conclusion is premature. First, if $C_{\mathbf{a}}$ vanishes for some values of $\mathbf{a}$, then these outcomes never occur, independent of the state being measured. Thus, the number of outcomes of the parent is only the number of nonvanishing POVM elements, which could be substantially smaller than $o^{m}$. Second, a given set of measurements does not uniquely determine a parent measurement. In general there are infinitely many parent measurements for a given set of compatible measurements. This is analogous to the fact that there are in general infinitely many joint probability distributions consistent with a set of marginal probabilities. Thus, even if for a given parent none of the POVM elements vanish, this does not rule out the possibility of there being another parent, such that some of its POVM elements vanish.

In order to understand how complex parent measurements are, we thus need to study the simplest possible parent for a given set of compatible measurements. More generally, this leads to a number of questions concerning the structure of the set of compatible measurements, which arise from the idea of studying the complexity of parent measurements. (i) Do there exist sets of compatible measurements such that the simplest parent necessarily has all elements nonvanishing? (ii) If not, then what is the dependence on the number of measurements $m$ in the worst case, i.e., is the complexity exponential in $m$ ? (iii) Which sets of measurements have the simplest parents and how simple are they (excluding trivial cases, such as when the measurements $\mathbb{M}_{x}$ are equal or a coarse graining of each other)? (iv) For typical sets of measurements, chosen at random with respect to a natural measure, are parents typically complex or simple?

In the remainder of this paper we present our findings concerning these questions. We believe that these questions, along with the many related ones that they lead to, collectively constitute an interesting line of investigation.

\section{FROM PARENT MEASUREMENTS TO COMPATIBLE CHILDREN}

It is instructive to start by focusing on parent measurements of the form (1) and their resulting children. Consider as a concrete example the parent measurement $\mathbb{K}$ with POVM elements

$$
K_{\lambda}=\frac{1}{3}\left(\mathbb{1}+\hat{\mathbf{n}}_{\lambda} \cdot \boldsymbol{\sigma}\right)
$$

for $\lambda=0,1,2$, where $\sigma=(X, Y, Z)$ is the vector of Pauli operators and $\hat{\mathbf{n}}_{\lambda}=(\cos (2 \lambda \pi / 3), \sin (2 \lambda \pi / 3), 0)$. Such a measurement, referred to as the trine, has outcomes corresponding to three vertices of an equilateral triangle in the $x y$ plane of the Bloch sphere. The trine measurement leads straightforwardly to a set of three compatible measurements $\mathbb{L}, \mathbb{M}$, and $\mathbb{N}$ via

$$
\begin{array}{lll}
L_{0}=K_{0}, & M_{0}=K_{1}, & N_{0}=K_{2}, \\
L_{1}=K_{1}+K_{2}, & M_{1}=K_{0}+K_{2}, & N_{1}=K_{0}+K_{1},
\end{array}
$$

which are just the three partitions of the three-outcome parent into two-outcome children. This constitutes a set of three two-outcome measurements that has a simple parent with only three outcomes. The complexity of such a parent is much simpler than that of the canonical parent that gives the exponential upper bound $2^{3}=8$ on the number of outcomes. Notice also that this moreover provides an example of a set of three measurements which are compatible, despite not commuting. 
This example can be straightforwardly generalized. It is always possible to form children from a parent by considering all of the partitions of the parent's outcomes. The total number of partitions of a set of size $o$ is the Bell number $B_{o}$ [9]. Discounting the two trivial partitions (the parent itself and the trivial measurement with a single outcome), a parent with $o$ outcomes is always a parent for a set of $B_{o}-2$ measurements. The Bell numbers grow rapidly, which has crucial consequences for the complexity of compatible measurements. Take for instance the case of a parent with six outcomes. Here $B_{6}=$ 203; hence every six-outcome measurement leads to a set of 201 measurements that are compatible. Therefore, these 201 compatible measurements arise from a six-outcome parent, which is overwhelmingly simpler than the canonical parent (upper bound), whose number of elements is exponential in the number of measurements.

\section{MAXIMALLY COMPLEX SETS OF COMPATIBLE MEASUREMENTS}

We now turn our attention to the other direction and study whether there exist sets of compatible measurements with the opposite behavior: to be maximally complex, i.e., such that the only possible parent POVM necessarily contains $o^{m}$ outcomes.

In the simplest case, of two measurements with two outcomes, it is straightforward to show that maximally complex sets of measurements exist. Consider noisy Pauli measurements

$$
M_{a}=\frac{1}{2}\left[\mathbb{1}+\eta(-1)^{a} X\right], \quad N_{b}=\frac{1}{2}\left[\mathbb{1}+\eta(-1)^{b} Z\right],
$$

where $a$ and $b$ are in $\{0,1\}$ and $X$ and $Z$ are the Pauli operators. Such measurements are compatible for $\eta \leqslant 1 / \sqrt{2}$. A parent measurement for $\eta=1 / \sqrt{2}$ is given by

$$
C_{a b}=\frac{1}{4}\left(\mathbb{1}+\frac{(-1)^{a} X+(-1)^{b} Z}{\sqrt{2}}\right) .
$$

This parent has four nonzero elements. Let us assume that there exists a parent with only three nonzero elements, e.g., $C_{00}=0$ (all other cases follow identically). In order to reproduce $M_{a}$ and $N_{b}$, we must have

$$
\begin{aligned}
M_{0} & =C_{00}+C_{01}=C_{01}, \\
N_{0} & =C_{00}+C_{10}=C_{10}
\end{aligned}
$$

and in order to be normalized we must have

$$
\mathbb{1}=C_{00}+C_{01}+C_{10}+C_{11}=M_{0}+N_{0}+C_{11} .
$$

This implies that

$$
C_{11}=\mathbb{1}-M_{0}-N_{0}=-\eta(X+Z) .
$$

This operator is however not positive semidefinite for any $\eta \neq$ 0 . Thus, there is no parent POVM with three elements for the set of measurements in Eq. (7).

The above example was particularly simple because there is a unique parent. In general, a parent has $o^{m}$ elements and there are $(o-1) m+1$ linearly independent constraints that these elements satisfy to reproduce the given measurements: a constraint from all but one of the POVM elements of each of the measurements $\mathbb{M}_{x}$, in addition to the normalisation constraint. The only situation with a unique parent is when $o^{m}-(o-1) m-1$ elements of the parent vanish, i.e., it has $(o-1) m+1$ outcomes. The above situation where $o=m=$ 2 is the only situation where $(o-1) m+1=o^{m}-1$ and a unique solution exists under the assumption that the parent is not maximally complex.

The next simplest case that can be considered is $m=3$ measurements with $o=2$ outcomes. Here we were able to find an example for $d=3$ (qutrit measurements). In particular,

$$
\begin{aligned}
L_{a} & =\frac{1}{2}\left[\mathbb{1}_{3}+(-1)^{a}\left(\frac{3 \sqrt{2}}{8} X_{01}+\frac{1}{2} X_{02}\right)\right], \\
M_{b} & =\frac{1}{2}\left[\mathbb{1}_{3}+(-1)^{b}\left(\frac{3 \sqrt{2}}{8} Z_{01}+\frac{1}{2} X_{12}\right)\right], \\
N_{c} & =\frac{1}{2}\left(\mathbb{1}_{3}+(-1)^{c} \frac{1}{2}\left(Z_{02}+Z_{12}\right)\right),
\end{aligned}
$$

where $a, b$, and $c$ are in $\{0,1,2\}$ and $X_{i j}=|i\rangle\langle j|+| j\rangle\langle i|$ and $Z_{i j}=|i\rangle\langle i|-| j\rangle\langle j|$ are Pauli operators acting on the subspace spanned by $|i\rangle$ and $|j\rangle$.

These measurements can be shown to be jointly measurable, with a parent with eight nonvanishing elements. It can also be shown that it is impossible to find a parent with seven or fewer nonvanishing elements. In particular, if $C_{a b c}=0$ is imposed, for any value of $a, b$, and $c$, then no parent exists for this set of measurements. The proof of this claim is not as straightforward as in the previous example and uses a method we developed, based upon duality theory of semidefinite programs, which we explain below. It allows us to find a certificate (or "witness"), which guarantees that there is no parent measurement if any POVM element of the parent vanishes. This shows that it is possible to certify conclusively that parents with given elements vanishing do not exist.

Finally, by a heuristic search method we were able to numerically find examples in a number of other cases: for two measurements $(m=2)$ in dimension $d$ with $o=d$ outcomes, requiring a parent with $d^{2}$ elements in each case. Details are provided in an accompanying online notebook [10].

\section{Method for lower bounding complexity}

In order to find parent POVMs which are not maximal, we can modify the SDP formulation of joint measurability to tackle the problem. In particular, if we define by $O(\mathbb{C})=\{\mathbf{a} \mid$ $\left.C_{\mathbf{a}} \neq 0\right\}$ the list of outcomes of $\mathbb{C}$, then we find that the joint measurability problem remains an SDP when we specify this list. That is, a parent POVM will exist with the only nonzero outcomes a subset of $O(\mathbb{C})$ if and only if the value of the following SDP is zero:

$$
\max _{v,\left\{C_{\mathbf{a}}\right\}} v
$$

subject to $M_{a \mid x}=\sum_{\mathbf{a} \in O(\mathbb{C})} D_{\mathbf{a}}(a \mid x) C_{\mathbf{a}} \forall a<(o-1), x$,

$$
\begin{aligned}
C_{\mathbf{a}} & \geqslant \nu \mathbb{1} \forall \mathbf{a} \in O(\mathbb{C}), \\
\mathbb{1} & =\sum_{\mathbf{a} \in O(\mathbb{C})} C_{\mathbf{a}}, \\
0 & \geqslant \nu .
\end{aligned}
$$


Here we recall that, due to normalization, it is not necessary to impose the final constraint (when $a=o-1$ ) on each element of $M_{a \mid x}$, as it is automatically satisfied. Note that, for later convenience, we have also moved away from the feasibility formulation of the problem, to a more standard maximization formulation, by relaxing the variables of the problem to be allowed to be not positive semidefinite and maximizing the minimal (negative) eigenvalue.

The benefit of this form of the problem over its feasibility version is that we can then use duality theory to find certificates (or witnesses) that a given set of compatible measurements has complexity at least $c$, by proving that the value of $(12)$ is strictly negative for all sets $O(\mathbb{C})$ of size $c-1$. That is, we can check all possible parents with a given number of elements and if none of them provide a valid parent POVM, then the complexity must be larger than the number checked.

We note that this requires the evaluation of $\left(c_{c}^{m}\right)$ semidefinite programs, a number which quickly becomes impractical. In the case of checking for maximal complexity, i.e., $c=$ $o^{m}-1$, it requires the evaluation of $o^{m}$ SDPs.

The dual formulation of the SDP of Eq. (12) is particularly useful, as we see next. Using standard techniques for obtaining the dual formulation of a convex optimisation problem, it can be shown that the dual formulation of (12) is given by

$$
\begin{aligned}
& \min _{\left\{\rho_{a x}\right\}, \omega} \operatorname{tr} \sum_{a \neq(o-1), x} \rho_{a x} M_{a \mid x}+\operatorname{tr} \omega \\
& \text { subject to } \omega+\sum_{a \neq(o-1), x} D_{\mathbf{a}}(a \mid x) \rho_{a x} \geqslant 0 \forall \mathbf{a} \in O(\mathbb{C}) \text {, } \\
& 1 \geqslant \operatorname{tr} \sum_{\substack{a \neq(o-1), x \\
\mathbf{a} \in O(\mathbb{C})}} D_{\mathbf{a}}(a \mid x) \rho_{a x}+o_{\mathbb{C}} \operatorname{tr} \omega,
\end{aligned}
$$

where $\left\{\rho_{a x}\right\}_{a, x}$ and $\omega$ are the dual variables and $o_{\mathbb{C}}=|O(\mathbb{C})|$. This dual optimization problem is useful due to weak duality, which says that for any feasible set of dual variables $\left\{\rho_{a x}\right\}_{a, x}$ and $\omega$,

$$
\operatorname{tr} \sum_{a \neq(o-1), x} \rho_{a x} M_{a \mid x}+\operatorname{tr} \omega \geqslant v^{*},
$$

where $v^{*}$ is the optimal value of (12). Note that $\left\{\rho_{a x}\right\}_{a, x}$ and $\omega$ do not even need to be optimal for the problem (13) for this to hold. Therefore, if we can find explicit choices of $\left\{\rho_{a x}\right\}_{a, x}$ and $\omega$ for each set $O(\mathbb{C})$ with $o_{\mathbb{C}}$ outcomes, such that $\operatorname{tr} \sum_{a \neq(o-1), x} \rho_{a x} M_{a \mid x}+\operatorname{tr} \omega<0$, then this provides a certificate, or witness, that the complexity of $\left\{\mathbb{M}_{x}\right\}_{x}$ is at least $o_{\mathbb{C}}+1$.

Next we will show that the maximal complexity of a set of measurements in fact does not grow exponentially, but much slower in the number of measurements $m$.

\section{BOUNDING THE COMPLEXITY OF COMPATIBILITY}

We now provide a proof that the number of outcomes of a parent measurement can always be bounded from above, by considering the geometry of the set of compatible measurements. We will demonstrate below that for any set of $m$ measurements $\left\{\mathbb{M}_{x}\right\}_{x}$, with $o$ outcomes in dimension $d$, a parent measurement can always be found which has no more than

$$
d^{2}[m(o-1)+1]
$$

outcomes. That is, the dependence of the complexity on the number of measurements is in fact linear and not exponential.

The basic idea behind the proof, which we give in full below, is to use the geometry of the set of compatible measurements. It has the structure of a cone, with extremal rays given by deterministic subnormalized measurements. Caratheodory's theorem [11] then shows that no more than $d^{2}[m(o-1)+1]$ extremal rays are necessary to form an arbitrary point inside the cone. Translated back, this means that a parent never needs more than this many outcomes.

Note that this bound can be larger than $o^{m}$, which in particular happens when $m=2$ and $o=d$ (as in the previous examples), hence why maximally complex sets can be found in certain cases. However, in general this shows that as the number of measurements grows, the complexity does not increase exponentially, as would be naively thought.

\section{Proof of upper bound}

Since we are considering $d$-dimensional measurements, each POVM element is specified by $d^{2}$ real parameters. Once $o-1$ POVM elements of a given measurement are specified, then the remaining element is fixed, due to the normalization condition $\sum_{a} M_{a \mid x}=\mathbb{1}$ for all $x$. Thus, in total $d^{2}[m(o-1)]$ real parameters are required to uniquely specify a set of measurements. In what follows we will also redundantly keep track of the right-hand side of the normalization condition, since we will need to relax it. That is, we will also keep track of the $d^{2}$ parameters necessary to specify $\mathbb{1}$. As such, we can represent a set of measurements by a point in a real vector space $\mathbb{R}^{D}$, where $D=d^{2}[m(o-1)+1]$.

Consider now a set of compatible measurements such that

$$
M_{a \mid x}=\sum_{\mathbf{a}} D_{\mathbf{a}}(a \mid x) C_{\mathbf{a}}
$$

For each a, we can also represent $D_{\mathbf{a}}(a \mid x) C_{\mathbf{a}}$ by a point in the same space $\mathbb{R}^{D}$. Note that for such points $\sum_{a} D_{\mathbf{a}}(a \mid x) C_{\mathbf{a}}=$ $C_{\mathrm{a}}$; thus these do not represent measurements, but rather submeasurements, since $C_{\mathbf{a}} \leqslant \mathbb{1}$. This was the reason for including the extra $d^{2}$ parameters in the above, since this allows us to consider the space of all submeasurements.

Geometrically, we see that the point in $\mathbb{R}^{D}$ corresponding to the collection of measurements $\left\{\mathbb{M}_{x}\right\}_{x}$ is a positive combination of at most $o^{m}$ points, one corresponding to each $D_{\mathbf{a}}(a \mid x) C_{\mathbf{a}}$ (since some of the $C_{\mathbf{a}}$ may vanish, such points correspond to the origin in $\mathbb{R}^{D}$ and all these points map degenerately there).

Assume now that a parent has been found with $k>D$ outcomes, i.e., such that $k$ of the $C_{\mathbf{a}}$ do not vanish. We thus have $k$ points in $D$ dimensions, and hence the points cannot all be linearly independent. Therefore, there must exist numbers $\lambda_{\mathbf{a}}$ such that

$$
\sum_{\mathbf{a}} \lambda_{\mathbf{a}} D_{\mathbf{a}}(a \mid x) C_{\mathbf{a}}=0 .
$$


We take $\lambda_{\mathbf{a}}=0$ for any a such that $C_{\mathbf{a}}=0$, such that the summation indeed runs over all values of $\mathbf{a}$ and not only the $k$ nonvanishing elements.

Therefore, we see that the following equation holds for all $\gamma$ :

$$
M_{a \mid x}=\sum_{\mathbf{a}}\left(1-\gamma \lambda_{\mathbf{a}}\right) D_{\mathbf{a}}(a \mid x) C_{\mathbf{a}} .
$$

This implies the existence of a new potential parent measurement $\mathbb{C}^{\prime}=\left\{C_{\mathbf{a}}^{\prime}\right\}_{\mathbf{a}}$, with

$$
C_{\mathbf{a}}^{\prime}=\left(1-\gamma \lambda_{\mathbf{a}}\right) C_{\mathbf{a}} .
$$

This will only be a valid measurement if $C_{\mathbf{a}}^{\prime} \geqslant 0$ for all $\mathbf{a}$. Therefore, we take

$$
\gamma=\frac{1}{\max _{\mathbf{a}} \lambda_{\mathbf{a}}}
$$

such that for all $\mathbf{a}$ it holds that $1-\gamma \lambda_{\mathbf{a}} \geqslant 0$ and for at least one value of $\mathbf{a}, 1-\gamma \lambda_{\mathbf{a}}=0$.

What this implies is that, starting from the assumption of a parent with $k$ nonvanishing outcomes, we have a procedure to obtain a parent with at most $k-1$ nonvanishing outcomes. This holds whenever $k>D$, since then we are always guaranteed that the points cannot be linearly independent. We can thus iterate this procedure until we are left with a parent with $D$ outcomes (or fewer, if in the last step multiple outcomes simultaneously vanish), at which point we can no longer guarantee linear dependence between the remaining POVM elements of the parent and must terminate the procedure.

This argument is nothing but Caratheodory's theorem for cones (presented in terms of submeasurements), which states that any point inside a convex cone in dimension $D$ can be written as a conic combination of at most $D$ extremal rays.

\section{MEMORY VERSUS TIME TRADE-OFF}

A major barrier to determining whether a set of measurements is compatible or not is the exponential increase in the size of the SDP optimization problem that has to be solved as the number of measurements increases. Since canonical parents have exponentially many outcomes in the number of measurements, it quickly becomes impractical, due to lack of memory, to determine whether a set of measurements is compatible or not.

The bound (15) however says that this exponential overhead is not required. Potentially the bound then implies that a more efficient algorithm could be found in order to determine whether a set of measurements is compatible or not.

Although this bound implies the existence of a much smaller parent, a new problem arises: to determine which elements of the canonical parent are nonvanishing. The bound implies that it is sufficient to check the $\left(\begin{array}{c}o^{n} \\ d^{2}[m(o-1)+1]\end{array}\right)$ parents with $d^{2}[m(o-1)+1]$ nonzero elements in order to determine whether the measurements are incompatible or not.

Thus the need for a large memory can be overcome, but at the expense of needing to carry out a significant number of calculations. In situations where calculations can easily be run in parallel, this may provide a practical way to attack problem instances that were previously infeasible due to memory requirements.

\section{COMPLEXITY OF TYPICAL MEASUREMENTS}

A further interesting question is to understand the behavior of typical sets of compatible measurements. In scenarios where the bound (15) is tight, we see immediately that typical sets of compatible measurements will be maximally complex. This follows from the geometry of the problem; Since typical measurements will lie in the interior of the set, they will need to be conic combinations of $d^{2}[m(o-1)+1]$ points and not fewer.

In scenarios where the bound (15) is not tight, the situation is much less clear. It is interesting to ask whether most sets of compatible measurements have simple parents or not. One problem with tackling this directly is the need to generate random instances of compatible measurements, according to some measure. Here we focus on the typical behavior of sets of measurements on the boundary of the set of compatible measurements. In this case, we use the following procedure to induce a random measure on the boundary, based upon the Haar measure on unitary matrices.

(i) Randomly generate $m$ unitary matrices $\left\{U_{x}\right\}$ according to the Haar measure, and from them define $m$ ideal von Neumann measurements $\left\{\left\{\Pi_{a \mid x}\right\}_{a}\right\}_{x}$, via their eigenvector decomposition. These sets of measurements will be incompatible with probability one.

(ii) Using the dual formulation of the SDP for compatibility, extract the dual variables, which geometrically define a random direction in the space of sets of measurements.

(iii) Find the set of compatible measurements $\left\{\mathbb{M}_{x}\right\}_{x}$ which is furthest in this direction. This problem is an SDP and moreover will always find a set of compatible measurements on the boundary of the set, due to convexity.

This method induces a measure on the boundary of compatible measurements, starting from the Haar measure on unitary matrices. Sampling from this distribution over compatible measurements, we then estimate the probability distribution over complexity in a number of cases which are numerically tractable. The full numerical results can be found in the accompanying online notebook [10]. A summary of the results are presented in Fig. 1. We find a rather complicated structure, in all cases a distribution of parent sizes, indicating that the boundary has a rich structure.

\section{COMPLEXITY OF EINSTEIN-PODOLSKY-ROSEN STEERING}

The above also imply results about the complexity of local-hidden-state (LHS) models in the context of EinsteinPodolsky-Rosen steering [12-14]. Einstein-Podolsky-Rosen steering is the nonlocal effect whereby measurements performed by Alice on half of an entangled quantum state "steer" the states of Bob at a distance in a way which cannot be explained by a simple causal model (known as the LHS model). In particular, if Alice and Bob share a state $\rho^{A B}$ and Alice performs a measurement $\mathbb{M}_{x}$, then upon obtaining outcome $a$ she steers Bob into the unnormalized 


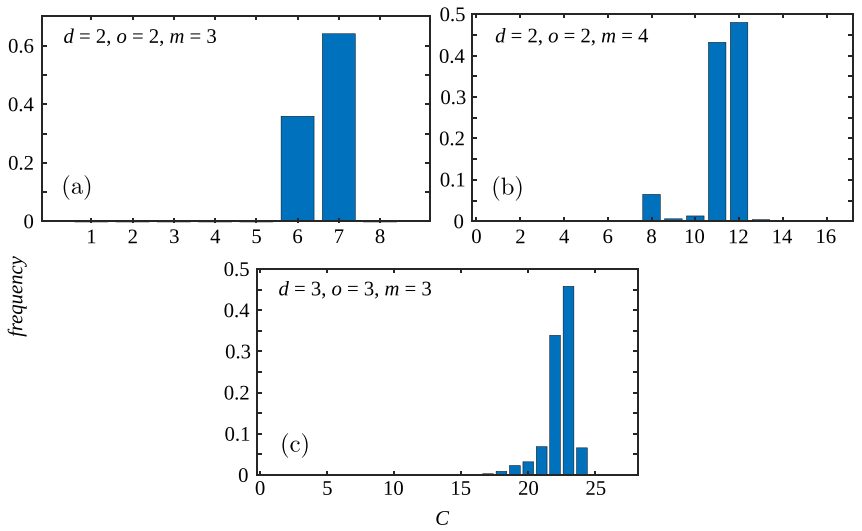

FIG. 1. Histograms showing the distribution of complexity for measurements sampled from the boundary of the jointly measurable set using the induced measure (as described in the main text). In each instance, 1000 points were sampled, and we give here the relative frequencies of the complexity of the measurements, computed numerically. Interestingly, in all cases, no maximally complex measurements are found.

state

$$
\sigma_{a \mid x}=\operatorname{tr}_{A}\left[\left(M_{a \mid x} \otimes \mathbb{1}\right) \rho^{\mathrm{AB}}\right] .
$$

The collection of states is said to have an LHS model if

$$
\sigma_{a \mid x}=\sum_{\lambda} p(\lambda) p(a \mid x, \lambda) \rho_{\lambda},
$$

where $\lambda$ is a classical hidden variable, distributed according to $p(\lambda), p(a \mid x, \lambda)$ are a collection of probabilities describing Alice's measurement outcome, and $\rho_{\lambda}$ are a collection of hidden normalized states, describing Bob's system.

Similarly to the case of compatibility, one can study the complexity of LHS models. First, a canonical form of LHS model can always be found, whereby

$$
\sigma_{a \mid x}=\sum_{\mathbf{a}} p(\mathbf{a}) D_{\mathbf{a}}(a \mid x) \rho_{\mathbf{a}}
$$

where now the hidden variable is the tuple $\mathbf{a}=\left(a_{1}, \ldots, a_{m}\right)$, which corresponds to a list of measurement results, one for each measurement of Alice, and $\rho_{\mathbf{a}}$ are the associated hidden states for Bob, which are jointly distributed according to $p(\mathbf{a})$. In this model, when Alice receives the hidden variable a and is asked to make the measurement $x$, she returns the measurement result $a=a_{x}$.

As in the case of compatible measurement, LHS models are inherently more complex than the assemblage of states they reproduce. In particular, the number of hidden states in the model is exponential in the number of measurement settings of Alice. We can thus ask, just as in the case of compatibility, whether it is always possible to find a simple LHS model, which contains only a small number of hidden states. In the following we will show that this is indeed the case, by exploiting the recently discovered connection between steering and measurement incompatibility $[15,16]$.

Using this connection, we will show that the maximal number of states needed in any canonical LHS model is the same as the number of elements in a canonical parent
POVM, i.e.,

$$
d^{2}[m(o-1)+1]
$$

The first step is to recap the one-to-one correspondence between incompatibility and steering that was recently found: Every set of measurements leads to steering if and only if it is incompatible $[15,16]$. In fact, starting from an LHS model in the steering scenario, it is always possible to obtain a parent measurement, and vice versa, using the following construction.

First, we note that the states in a steering scenario satisfy the no-signaling constraint $\sum_{a} \sigma_{a \mid x}=\rho$, where $\rho$ is the reduced density operator of Bob, which is independent of $x$. We consider the purification of this state

$$
|\psi\rangle=\sum_{i} \sqrt{\lambda_{i}}\left|\lambda_{i}\right\rangle\left|\lambda_{i}\right\rangle,
$$

where $\rho=\sum_{i} \lambda_{i}\left|\lambda_{i}\right\rangle\left\langle\lambda_{i}\right|$ is the diagonal form of Bob's reduced state. We then note that the operators form a collection of POVMs

$$
M_{a \mid x}=\sqrt{\rho^{-1}}\left(\sigma_{a \mid x}\right)^{\top} \sqrt{\rho^{-1}},
$$

where $T$ denotes transpose in the basis $\left\{\left|\lambda_{i}\right\rangle\right\}$, and if Alice and Bob share the state $|\psi\rangle$ (i.e., the purifying system is given to Alice) and she performs these POVMs, then this prepares the assemblage $\sigma_{a \mid x}$ for Bob

$$
\operatorname{tr}_{A}\left\{\left[\sqrt{\rho^{-1}}\left(\sigma_{a \mid x}\right)^{\top} \sqrt{\rho^{-1}} \otimes \mathbb{1}\right]|\psi\rangle\langle\psi|\right\}=\sigma_{a \mid x} .
$$

Equation (26) is the key equation for the one-to-one correspondence. Assume first that the assemblage $\sigma_{a \mid x}$ has an LHS model of the form (23); then the associated set of measurements has the form

$$
\begin{aligned}
M_{a \mid x} & =\sqrt{\rho^{-1}}\left(\sum_{\mathbf{a}} p(\mathbf{a}) D_{\mathbf{a}}(a \mid x) \rho_{\mathbf{a}}^{\top}\right) \sqrt{\rho^{-1}} \\
& =\sum_{\mathbf{a}} D_{\mathbf{a}}(a \mid x)\left[p(\mathbf{a}) \sqrt{\rho^{-1}} \rho_{\mathbf{a}}^{\top} \sqrt{\rho^{-1}}\right] .
\end{aligned}
$$

Defining $C_{\mathbf{a}}=p(\mathbf{a}) \sqrt{\rho^{-1}} \rho_{\mathbf{a}}^{\top} \sqrt{\rho^{-1}}$, which is positive semidefinite by construction and sums to the identity operator, we see that they constitute a canonical parent for the measurements $M_{a \mid x}$. In the other direction, the calculation follows identically. In particular, from (26) it follows that

$$
\sigma_{a \mid x}=\sqrt{\rho} M_{a \mid x}^{\top} \sqrt{\rho} .
$$

Hence, if the $M_{a \mid x}$ form a set of compatible measurements, with the parent satisfying $M_{a \mid x}=\sum_{\mathbf{a}} D_{\mathbf{a}}(a \mid x) C_{\mathbf{a}}$, then

$$
\sigma_{a \mid x}=\sum_{\mathbf{a}} D_{\mathbf{a}}(a \mid x) \sqrt{\rho} C_{\mathbf{a}}^{\top} \sqrt{\rho} .
$$

Defining $\quad p(\mathbf{a})=\operatorname{tr}\left[\sqrt{\rho} C_{\mathbf{a}}^{\top} \sqrt{\rho}\right]$ and $\rho_{\mathbf{a}}=\sqrt{\rho} C_{\mathbf{a}}^{\top} \sqrt{\rho} / p(\mathbf{a})$, which are seen to correspond to a valid probability distribution 
and a set of normalized quantum states, we thus recover an LHS model for the assemblage $\sigma_{a \mid x}$.

Thus, putting everything together, whenever we have an assemblage that has an LHS model, we can always find a set of compatible measurements that reproduce the assemblage. The correspondence furthermore shows that the LHS model and the parent measurement are directly related to each other. Hence, our construction for finding a parent measurement with at most $d^{2}[o(m-1)+1]$ outcomes directly implies the existence of an LHS model with at most this many hidden states.

\section{PROBABILISTIC PARENTS}

We finally discuss the possibility of a more general class of parent measurements and the implications this may have for complexity. When introducing joint measurability, our general definition was that children are generated from parents by partitioning the outcomes of the parent. A slightly more general definition is to introduce randomness and allow for mixing over partitions. In this case, the pair of measurements $\mathbb{M}=\left\{M_{a}\right\}_{a}$ and $\mathbb{N}=\left\{N_{b}\right\}_{b}$ are jointly measurable if there exist a parent measurement $\mathbb{K}=\left\{K_{\lambda}\right\}_{\lambda}$ and a pair of conditional probability distributions $p_{\mathbb{M}}(a \mid \lambda)$ and $p_{\mathbb{N}}(b \mid \lambda)$ such that

$$
M_{a}=\sum_{\lambda} p_{\mathbb{M}}(a \mid \lambda) K_{\lambda}, \quad N_{b}=\sum_{\lambda} p_{\mathbb{N}}(b \mid \lambda) K_{\lambda} .
$$

This definition does not change whether or not a set of measurements is compatible with respect to our previous notion, since it is always possible to go from this form to the canonical form [17]. It also does not affect our upper bound (15), since this provides an explicit parent for a set of children. It does however have implications for the existence of maximally complex sets of compatible measurements, since in principle it might be possible to reduce the size of the parent by using randomness.

We find that this is indeed the case. For the example given in Eq. (7) we were able to find the probabilistic parent with only three outcomes

$$
K_{0}=\frac{2 M_{0}-\eta \sqrt{2} N_{0}}{2-\eta^{2}}, \quad K_{1}=\frac{2 N_{0}-\eta \sqrt{2} M_{0}}{2-\eta^{2}},
$$

and $K_{2}=\mathbb{1}-K_{0}-K_{1}$. Indeed, $\left\{K_{1}, K_{2}, K_{3}\right\}$ is a valid POVM for $\eta \lesssim 0.5609$ and reproduces $\mathbb{M}$ and $\mathbb{N}$ with

$$
M_{0}=K_{0}+\frac{\eta}{\sqrt{2}} K_{1}, \quad N_{0}=\frac{\eta}{\sqrt{2}} K_{0}+K_{1} .
$$

We do not know whether or not a probabilistic parent exists for larger values of $\eta \leqslant 1 / \sqrt{2}$. Nevertheless, no deterministic parent with three outcomes exists for any $\eta$, as previously shown.

The nature of the problem of finding probabilistic parents seems much richer than the problem of finding deterministic parents, which has the simple form of a convex optimization problem. When introducing probabilistic parents, this convex structure is lost and it seems much harder to decide whether a probabilistic parent of a given size exists. Furthermore, this raises an interesting question regarding the possible trade-off between the amount of randomness necessary and the size of the parent. We leave further exploration of these interesting questions for future work.

\section{CONCLUSION}

In this work we have instigated the study of the complexity of a set of compatible measurements, in terms of how many outcomes their parent measurement necessarily has. We have shown in one direction that very large sets of compatible measurements can be formed starting from a simple parent (i.e., one with only a few outcomes) and in the other direction that the complexity of compatibility can be bounded and scales no worse than linearly in the number of measurements $m$. We have also explored the typical behavior of the boundary of compatible measurements in instances where this bound is not tight and shown that in these cases the boundary appears to have a rich structure. We have finally raised the possibility of using randomness in the parent and found examples where this reduces the number of outcomes of the parent. This raises interesting and subtle questions regarding what this implies for complexity. We leave this investigation for future work.

We believe our results raise many interesting questions about the structure of compatible measurements and potentially suggests a way to quantify "how compatible" a set of measurements are. We hope that it will lead to further exciting work in this and related directions.

\section{ACKNOWLEDGMENTS}

P.S. acknowledges support from a Royal Society URF (UHQT). A.B.S. acknowledges support from the Foundation for Polish Science (IRAP project, ICTQT, Contract No. 2018/MAB/5, cofinanced by the EU within Smart Growth Operational Programme). This research was partially supported by Perimeter Institute for Theoretical Physics. Research at Perimeter Institute was supported by the Government of Canada through the Department of Innovation, Science and Economic Development Canada and by the Province of Ontario through the Ministry of Research, Innovation and Science. M.J.H. and A.B.S. acknowledge the FQXi large grant "The Emergence of Agents from Causal Order".

\section{APPENDIX: CERTIFICATE FOR $\boldsymbol{m}=3$ MEASUREMENTS WITH $o=2$ OUTCOMES}

In this Appendix we apply the method given in Sec. V to prove that the example (11) (with $m=3$ measurements and $o=2$ outcomes) requires a maximal parent with eight outcomes. The set of measurements defined in Eq. (11) may be expressed as follows, using a slightly more general notation which will be useful later:

$$
\begin{aligned}
& M_{a_{0} \mid 0}=\frac{1}{2}\left[\mathbb{1}_{3}+(-1)^{a_{0}}\left(\frac{3 \sqrt{2}}{8} X_{01}+\frac{1}{2} X_{02}\right)\right], \\
& M_{a_{1} \mid 1}=\frac{1}{2}\left[\mathbb{1}_{3}+(-1)^{a_{1}}\left(\frac{3 \sqrt{2}}{8} Z_{01}+\frac{1}{2} X_{12}\right)\right], \\
& M_{a_{2} \mid 2}=\frac{1}{2}\left(\mathbb{1}_{3}+(-1)^{a_{2}} \frac{1}{2}\left(Z_{02}+Z_{12}\right)\right) .
\end{aligned}
$$


Here $X_{i j}=|i\rangle\langle j|+| j\rangle\langle i|$ and $Z_{i j}=|i\rangle\langle i|-| j\rangle\langle j|$ are Pauli operators acting on the subspace spanned by $|i\rangle$ and $|j\rangle$. These measurements are jointly measurable, and a parent with eight elements is given by $C_{a_{0} a_{1} a_{2}}=\frac{3}{8}\left|\phi_{a_{0} a_{1} a_{2}}\right\rangle\left\langle\phi_{a_{0} a_{1} a_{2}}\right|$, where

$$
\begin{aligned}
\left|\phi_{a_{0} a_{1} 0}\right\rangle & =(-1)^{a_{0}} \cos \frac{\pi}{8}\left|a_{1}\right\rangle+\sin \frac{\pi}{8}\left|a_{1} \oplus 1\right\rangle, \\
\left|\phi_{a_{0} a_{1} 1}\right\rangle & =\frac{|0\rangle+(-1)^{\left(a_{0}+a_{1}\right)}|1\rangle+2(-1)^{a_{0}}|2\rangle}{\sqrt{6}} .
\end{aligned}
$$

Here we will show that it is not possible to find a parent with seven or fewer nonvanishing elements.

Let us assume that there is a parent with seven elements and denote by $\mathbf{a}^{*}$ the string of outcomes corresponding to the POVM element of the parent which is assumed to vanish, i.e., $C_{\mathbf{a}^{*}}=0$. Consider the sets of dual variables

$$
\begin{gathered}
\rho_{0 x}^{\mathbf{a}^{*}}=\frac{1}{5}(-1)^{a_{x}^{*}}\left|\psi_{\mathbf{a}^{*}}\right\rangle\left\langle\psi_{\mathbf{a}^{*}}\right|, \\
\omega^{\mathbf{a}^{*}}=\frac{1}{5}\left[\left(\bar{a}_{0}^{*}+\bar{a}_{1}^{*}\right) \bar{a}_{2}^{*}+\left(\bar{a}_{0}^{*}+\bar{a}_{1}^{*}-1\right) \bar{a}_{2}^{*}\right]\left|\psi_{\mathbf{a}^{*}}\right\rangle\left\langle\psi_{\mathbf{a}^{*}}\right|,
\end{gathered}
$$

where $\bar{a}_{x}^{*} \equiv a_{x}^{*} \oplus 1$,

$$
\begin{aligned}
\left|\psi_{\mathbf{a}^{*}}\right\rangle= & (-1)^{\left(a_{0}^{*}+a_{1}^{*}\right)} \alpha_{a_{2}^{*}}\left|a_{1}^{*}\right\rangle+\beta_{a_{2}^{*}}\left|a_{1}^{*} \oplus 1\right\rangle \\
& +(-1)^{a_{0}^{*} a_{1}^{*}} \gamma_{a_{2}^{*}}|2\rangle,
\end{aligned}
$$

and $\left(\alpha_{a_{2}^{*}}, \beta_{a_{2}^{*}}, \gamma_{a_{2}^{*}}\right)$ are amplitudes which will be specified shortly. Direct substitution shows that for any choice of $\left(\alpha_{a_{2}^{*}}, \beta_{a_{2}^{*}}, \gamma_{a_{2}^{*}}\right)$, for all $\mathbf{a}^{*}$,

$$
\begin{array}{r}
\omega^{\mathbf{a}^{*}}+\sum_{x} D_{\mathbf{a}}(0 \mid x) \rho_{0 x}^{\mathbf{a}^{*}} \geqslant 0 \forall \mathbf{a} \neq \mathbf{a}^{*}, \\
1 \geqslant \operatorname{tr} \sum_{x, \mathbf{a} \neq \mathbf{a}^{*}} D_{\mathbf{a}}(0 \mid x) \rho_{0 x}^{\mathbf{a}^{*}}+7 \operatorname{tr} \omega^{\mathbf{a}^{*}},
\end{array}
$$

which shows that $\rho_{0 x}^{\mathbf{a}^{*}}$ and $\omega^{\mathbf{a}^{*}}$ are feasible solutions for the dual problem (13).

Choosing

$$
\left(\alpha_{a_{2}^{*}}, \beta_{a_{2}^{*}}, \gamma_{a_{2}^{*}}\right)= \begin{cases}(1,0,0) & \text { if } a_{2}^{*}=0 \\ \left(\frac{\sqrt{2}}{4}, \frac{\sqrt{2}}{4}, \frac{\sqrt{3}}{2}\right) & \text { if } a_{2}^{*}=1\end{cases}
$$

leads to

$$
\operatorname{tr} \sum_{x} \rho_{0 x}^{\mathbf{a}^{*}} M_{a \mid x}+\operatorname{tr} \omega^{\mathbf{a}^{*}}= \begin{cases}\frac{4-3 \sqrt{2}}{80} & \text { if } a_{2}^{*}=0 \\ \frac{12-8 \sqrt{6}-(-1)^{\mathbf{a}_{1}^{*}} 3 \sqrt{2}}{320} & \text { if } a_{2}^{*}=1,\end{cases}
$$

which is negative in all cases. This provides the required proof that it is impossible to find a parent for the set of measurements (A1) with seven or fewer elements. In particular, we have demonstrated a feasible solution to the dual problem that obtains a negative value, which implies that the solution of the primal problem is negative (and hence no parent exists). Our construction works for all eight choices of parents with (at least) one element vanishing.
[1] W. Heisenberg, Z. Phys. 43, 172 (1927).

[2] P. Busch, P. J. Lahti, and P. Mittelstaedt, The Quantum Theory of Measurement, 2nd ed., Lecture Notes in Physics Monographs (Springer, Berlin, 1996), Vol. 2.

[3] T. Heinosaari, T. Miyadera, and M. Ziman, J. Phys. A: Math. Theor. 49, 123001 (2016).

[4] E. Haapasalo, T. Heinosaari, and J.-P. Pellonpää, Rev. Math. Phys. 26, 1450002 (2014).

[5] L. Guerini and M. Terra Cunha, J. Math. Phys. 59, 042106 (2018).

[6] G. Sentís, B. Gendra, S. D. Bartlett, and A. C. Doherty, J. Phys. A: Math. Theor. 46, 375302 (2013).

[7] M. Oszmaniec, L. Guerini, P. Wittek, and A. Acín, Phys. Rev. Lett. 119, 190501 (2017).

[8] S. Boyd and L. Vandenberghe, Convex Optimization (Cambridge University Press, New York, 2004).

[9] E. T. Bell, Am. Math. Mon. 41, 411 (1934).
[10] The accompanying Jupyter Notebook can be found at https: //nbviewer.jupyter.org/github/paulskrzypczyk/complexityofcompatibility/blob/master/complexity-of-compatible-meas. ipynb.

[11] C. Carathéodory, Math. Ann. 64, 95 (1907).

[12] H. M. Wiseman, S. J. Jones, and A. C. Doherty, Phys. Rev. Lett. 98, 140402 (2007).

[13] D. Cavalcanti and P. Skrzypczyk, Rep. Prog. Phys. 80, 024001 (2017).

[14] R. Uola, A. C. S. Costa, H. C. Nguyen, and O. Gühne, Rev. Mod. Phys. 92, 015001 (2020).

[15] M. T. Quintino, T. Vértesi, and N. Brunner, Phys. Rev. Lett. 113, 160402 (2014)

[16] R. Uola, T. Moroder, and O. Gühne, Phys. Rev. Lett. 113, 160403 (2014).

[17] S. T. Ali, C. Carmeli, T. Heinosaari, and A. Toigo, Found. Phys. 39, 593 (2009). 